\title{
The equation of the oscillatory motion of the blade of the earth-moving machine depending on the properties of the ground and the parameters of the blade
}

\author{
Minsur Zemdikhanov ${ }^{1 *}{ }^{[0000-001-5207-2346]}$, Rustem Sakhapov ${ }^{10000-0001-9665-1251]}$, and Ramil \\ Gainutdinov ${ }^{1}$ \\ ${ }^{1}$ Kazan State University of Architecture and Engineering, 420043, Zelenaya st., Kazan, Russia
}

\begin{abstract}
The influence of the design parameters and technological properties of the ground on the nature of the oscillatory movement of the blade of the working body of the earth-moving machine is investigated.

The purpose of the study is to identify the force factors of the interaction of the oscillatory working body of the earth -moving machine with the ground and determine its optimal parameters.

The equation of the oscillatory motion of the blade of the working body of the earthmoving machine depending on its design parameters and technological properties of the ground is obtained.

The results can be used in the development and determination of the optimal design parameters of the working bodies of earth-moving machines.

Keywords: working body, oscillatory motion, knife, ground moving.
\end{abstract}

\section{Introduction}

You can increase the productivity of earth-moving machines by increasing the engine power and traction force. Another direction of increasing the productivity of these types of machines is to improve the scheme and design of their working bodies. This will allow, without increasing the power and traction of these types of machines, to increase its productivity by reducing the resistance of the ground to the working body of the earthmoving machine [1-4].

When carrying out earth-moving operations in order to reduce the resistance of the ground to the working bodies of machines, other methods can also be used. For example, based on the use of vibrations or vibrations of the working body of machines (active working bodies), as well as a shock or explosion [5].

Vibration organs include those working bodies on which vibration sources are installed, which inform the working body of vibrations of a certain frequency and in a certain direction. The resulting pulse load destroys the ground, thereby reducing the resistance of the working body, and therefore the machine $[6,7]$.

\footnotetext{
* Corresponding author: mr.minsur@mail.ru
} 
In earth-moving machines with blade and dump working bodies, vibration is usually transmitted to the dump or blade of the working body of the machine.

The disadvantage of vibrating working bodies of earth-moving machines is the complexity of the design, and sometimes a higher total energy consumption [8].

In this regard, the working bodies of machines are relevant, in which the oscillatory movement of the blade or cutting edge occurs due to the heterogeneity of the ground structure. Due to the heterogeneity of the ground structure, the resistance forces to the working body of the machine will also be variable. As a result, when interacting with the ground, there is a self-oscillating movement of the working body. Self-oscillating working bodies, unlike working bodies with an active drive, do not require energy supply, therefore, they have a simpler design and lower energy consumption. At the same time, they have all the advantages of working bodies with an active drive [9-11].

The purpose of the study is to determine the influence of the technological properties of the ground and the design parameters of the blade of the working body of the earth-moving machine on the nature of its oscillatory movement.

\section{Methods}

The scheme of the proposed self-oscillating working body of the earth-moving machine in the plan is shown in Fig. 1. The angle in the plan between the direction of movement and the line of the cutting edge of the blade when working is $\gamma_{0}$. The blade has the ability to rotate around the vertical axis $O Z$, passing through the tip of the blade (hinge A) and perpendicular to the plane of the drawing. Also, the blade 1 is spring-loaded with a spring 3 , which also in a special case can be made in the form of a torsion shaft also passing through the $O Z$ axis.

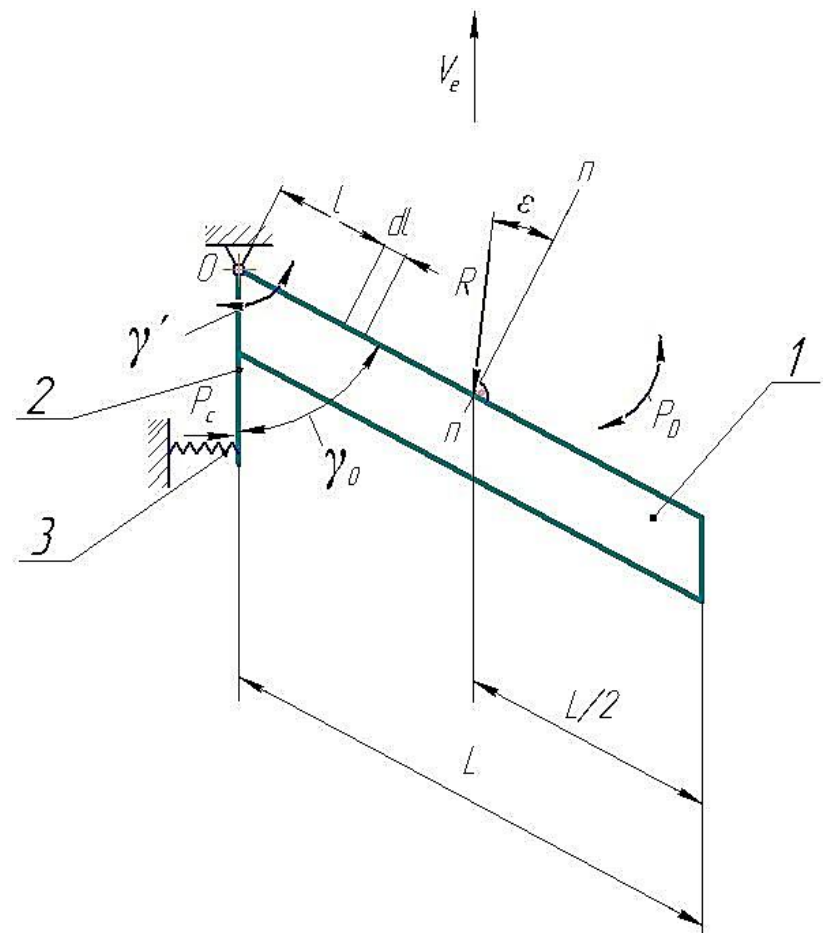

Fig. 1. Diagram of the forces acting on the blade of the working body. 
Having considered the process of interaction of the blade of the working body with the ground and determining the influence of its parameters on the parameters of self-oscillatory motion, we can describe the equation of its oscillatory motion.

When obtaining the equation, you can consider two options for attaching a blade that performs an oscillatory movement. The first option - the axis of rotation of the blade passes through the leftmost tip of the cutting edge of the blade, as shown in Fig. 1. The second option - the axis of rotation of the blade is located at some distance from the line of the cutting edge of the blade. In this study, we will consider the first option.

In this paper, the oscillatory motion of the blade of the working body relative to the vertical axis $O Z$, which also performs translational motion in the ground at a speed of $V_{\mathrm{e}}$, is theoretically investigated (Fig. 1).

Also, Fig. 1 shows the forces acting on the blade of the working body. The cutting edge 1 is limited from turning by the spring 3 interacting through the lever 2 . In Fig. 1, the force $R$, is the horizontal projection of the main vector of the ground resistance force on the direction of the forward movement of the blade. The moment $M_{R}$ of the force $R$ is balanced by the moment $M_{C}$, the force $P_{C}$, acting on the blade lever from the side of the spring 2 .

\section{Results and discussion}

The above diagram of the blade attachment requires the use of a spring 3 with high rigidity to balance the moment $M_{C}$. In this case, the self-oscillating system will have a high natural frequency of oscillations, determined by the formula:

$$
\omega_{0}=\frac{1}{2 \pi} \sqrt{\frac{c}{J}}
$$

where $c$ is the torsional rigidity of the blade turning system, $\mathrm{H} \mathrm{m} / \mathrm{rad}$; $J$ is the moment of inertia of the blade with the axis and lever relative to the vertical axis $O Z, \mathrm{~kg}^{*} \mathrm{~m}^{\wedge} 2$.

With the forward movement of the working body, under the action of the constant component of the resistance, the blade will be set at an angle $\gamma_{0}$, at which the moments $M_{R}$ and $M_{C}$ are mutually balanced. When the ground resistance changes, therefore, the value of $M_{R}$, the blade 1 performs an oscillatory movement at a certain angle $\gamma$ relative to the position with an angle $\gamma_{0}$. The energy of the oscillatory motion decreases due to friction losses. However, the inconstancy of the ground resistance force leads to the constant occurrence of new vibrations with different amplitudes.

In this design, the frequency of natural vibrations of the working body can be adjusted by changing the torsional rigidity of the system. For example, changing the spring stiffness or using springs with different characteristics.

The blade is also affected by the moment $M_{D}$ from the dissipative forces $P_{D}$ of the ground, which counteracts the oscillatory movement of the blade. Then the oscillatory motion of the blade of the working body of the machine can be described by the following equation:

$$
J \ddot{\gamma}=M_{R}+M_{C}+M_{D}
$$

where $M_{D}$ is the total moment of dissipative forces $P_{D}$.

The moment $M_{R}$ of the force $R$ is determined by the equation (Fig. 1):

$$
M_{R}=R \frac{L}{2} \cos \varepsilon,
$$

where $L$ is the length of the cutting edge of the blade; $\varepsilon$ is the angle of friction of the ground against the cutting edge of the blade during the translational movement of the working body.

The force $R$ can be expressed by the formula: 


$$
R=\frac{R_{Y}}{\sin \left(\varepsilon+\gamma_{0}\right)}
$$

where $R_{Y}$ is the projection of the force $R$ on the direction of movement of the working body; $\gamma_{0}$ is the angle in plan between the direction of translational movement of the working body and the line of the cutting edge of the blade, when $M_{R}$ and $M_{C}$ are mutually balanced and are respectively equal to $M_{R}{ }^{0}$ and $M_{C}{ }^{0}$.

The force of $R_{Y}$ is written in the following form [12]:

$$
R_{Y}=k_{0} B_{0}=k_{0} L \sin \gamma_{0}
$$

where $k_{0}$ is the specific resistance of the ground in the direction of movement of the working body, per unit width of the blade grip, $\mathrm{N} / \mathrm{m} ; B_{0}$ is the width of the blade grip, when the angle between the direction of movement of the working body and the line of the cutting edge of the blade is $\gamma_{0}$.

Substituting $R_{Y}$ from equation (5) into (4), we obtain:

$$
R=\frac{k_{0} L \sin \gamma_{0}}{\sin \left(\varepsilon+\gamma_{0}\right)}
$$

From equations (3) and (6), we obtain the following equation for determining $M_{R}{ }^{0}$ :

$$
M_{R}{ }^{0}=\frac{k_{0} L^{2} \cos \varepsilon \sin \gamma_{0}}{2 \sin \left(\varepsilon+\gamma_{0}\right)}
$$

Given that when the angle between the direction of movement of the working body and the line of the cutting edge of the blade is $\gamma_{0}$, then $M_{R}{ }^{0}=-M_{C}{ }^{0}$, we write:

$$
M_{C}{ }^{0}=-\frac{k_{0} L^{2} \cos \varepsilon \sin \gamma_{0}}{2 \sin \left(\varepsilon+\gamma_{0}\right)} \text {. }
$$

When the blade moves forward, it performs an self-oscillatory movement, deviating from the angle $\gamma_{0}$ by a certain angle $\gamma$, then the moments $M_{R}$ and $M_{C}$ are determined by the equations:

$$
\begin{gathered}
M_{R}=\frac{k_{0} L^{2} \cos \varepsilon \sin \left(\gamma_{0}+\gamma\right)}{2 \sin \left(\varepsilon+\gamma_{0}+\gamma\right)} \\
M_{C}=-\frac{k_{0} L^{2} \cos \varepsilon \sin \gamma_{0}}{2 \sin \left(\varepsilon+\gamma_{0}\right)}+c \gamma
\end{gathered}
$$

The energy loss of the oscillatory movement of the blade of the working body in the ground is determined, taking into account the dry, viscous and quadratic component of the friction force. The main moment of the dissipative friction forces is a quadratic dependence on the angular velocity and is directed against the direction of rotation of the blade [13-15]:

$$
M_{D}=\left(b_{1} m_{1} \operatorname{sgn} \dot{\gamma}+\dot{\gamma}+b_{3} m_{3} \dot{\gamma}^{2} \operatorname{sgn} \dot{\gamma}\right),
$$

where $b_{1}, b_{2}, b_{3}$ are coefficients that depend on the properties of the soil and relate to the dry, viscous and quadratic components of the friction force, respectively; $m_{1}, m_{2}, m_{3}$ are coefficients determined by the design parameters of the working body; - the Kronecker function, respectively, having the values $-1,0,1$ at $\dot{\gamma}<0, \dot{\gamma}=0, \dot{\gamma}>0[16,17]$. 
We determine the coefficients $m_{1}, m_{2}, m_{3}$ included in equation (11), which depend on the design parameters of the working body. The main part of the ground friction force on the working body of the machine acting on the blade, as is known, falls on its cutting edge. A much smaller part of the ground friction force affects the upper surface of the blade [18, 19].

Next, we write the formula (11) in the form:

$$
M_{D}=\left(M_{1} \operatorname{sgn} \dot{\gamma}+M_{2}+M_{3} \operatorname{sgn} \dot{\gamma}\right),
$$

where $M_{1}, M_{2}, M_{3}$ are the moments of ground resistance relative to the axis of rotation of the blade, created by the forces of dry, viscous and quadratic friction.

Next, we define the moments $M_{1}, M_{2}, M_{3}$ in the form of integrals:

$$
\begin{gathered}
M_{1}=b_{1} \int_{0}^{L} l d l=b_{1} \frac{L^{2}}{2} \\
M_{2}=b_{2} \int_{0}^{L} v_{l} l d l=b_{2} \dot{\gamma} \int_{0}^{L} l^{2} d l=b_{2} \frac{L^{3}}{3} \dot{\gamma} \\
M_{3}=b_{3} v_{l}^{2} l d l=b_{3} \dot{\gamma}^{2} \int_{0}^{L} l^{3} d l=b_{3} \frac{L^{4}}{4} \dot{\gamma}^{2}
\end{gathered}
$$

where $l$ is the distance from the axis of rotation of the blade to the segment $d l$ on the cutting edge of the blade; $v_{l}$ is the speed of the segment of the cutting edge $d l$ during the selfoscillating movement of the knife.

Then $m_{1}, m_{2}, m_{3}$ are determined by the following formulas:

$$
m_{1}=\frac{L^{2}}{2} ; \quad m_{2}=\frac{L^{3}}{3} ; \quad m_{3}=\frac{L^{4}}{4} .
$$

After the transformations, the equation of the self-oscillating motion of the blade of the working body (2) will take the form:

$$
J \ddot{\gamma}=\frac{k_{0} L^{2} \cos \varepsilon}{2}\left[\frac{\sin \left(\gamma_{0}+\gamma\right)}{\sin \left(\varepsilon+\gamma_{0}+\gamma\right)}-\frac{\sin \gamma_{0}}{\sin \left(\varepsilon+\gamma_{0}\right)}\right]+c \gamma-\left(b_{1} \frac{L^{2}}{2} \operatorname{sgn} \dot{\gamma}+b_{2} \frac{L^{3}}{3} \dot{\gamma}+b_{3} \frac{L^{4}}{4} \dot{\gamma}^{2} \operatorname{sgn} \dot{\gamma}\right)
$$

or, by entering the appropriate notation, we get:

$$
\ddot{\gamma}-A_{0}\left[\frac{\sin \left(\gamma_{0}+\gamma\right)}{\sin \left(\varepsilon+\gamma_{0}+\gamma\right)}-\frac{\sin \gamma_{0}}{\sin \left(\varepsilon+\gamma_{0}\right)}\right]-A_{c} \gamma+A_{2} \dot{\gamma}+\left(A_{1}+A_{3} \dot{\gamma}^{2}\right) \operatorname{sgn} \dot{\gamma}=0
$$

where $-A_{0}=\frac{k_{0} L^{2} \cos \varepsilon}{2 J} ; A_{C}=\frac{c}{J} ; A_{1}=\frac{b_{1} L^{2}}{2 J} ; A_{2}=\frac{b_{2} L^{3}}{3 J} ; A_{3}=\frac{b_{3} L^{4}}{4 J}$.

To determine the influence of the parameters of the working body on the nature of oscillations, equation (18) is numerically solved.

To solve these equations, it is necessary to determine the parameters that depend on the properties of the ground. The characteristics of the ground resistance were determined as a result of processing experimentally obtained vibrograms recorded during the movement of the working body with certain parameters in this ground. 
The parameters of the working body and the properties of the ground affect such characteristics of the movement of the working body as the frequency and period of vibrations and the attenuation decrement.

The results of the calculations are shown in Figs 2 and 3 in the form of graphs.

Fig. 2 shows the calculated vibrogram of the oscillatory motion of the working body having the parameters: $B_{0}=0,25 \mathrm{~m}, \gamma_{0}=45^{0}, J=0,04 \mathrm{~kg} * \mathrm{~m}^{\wedge} 2, c=45 \mathrm{H} \mathrm{m} /$ grad.

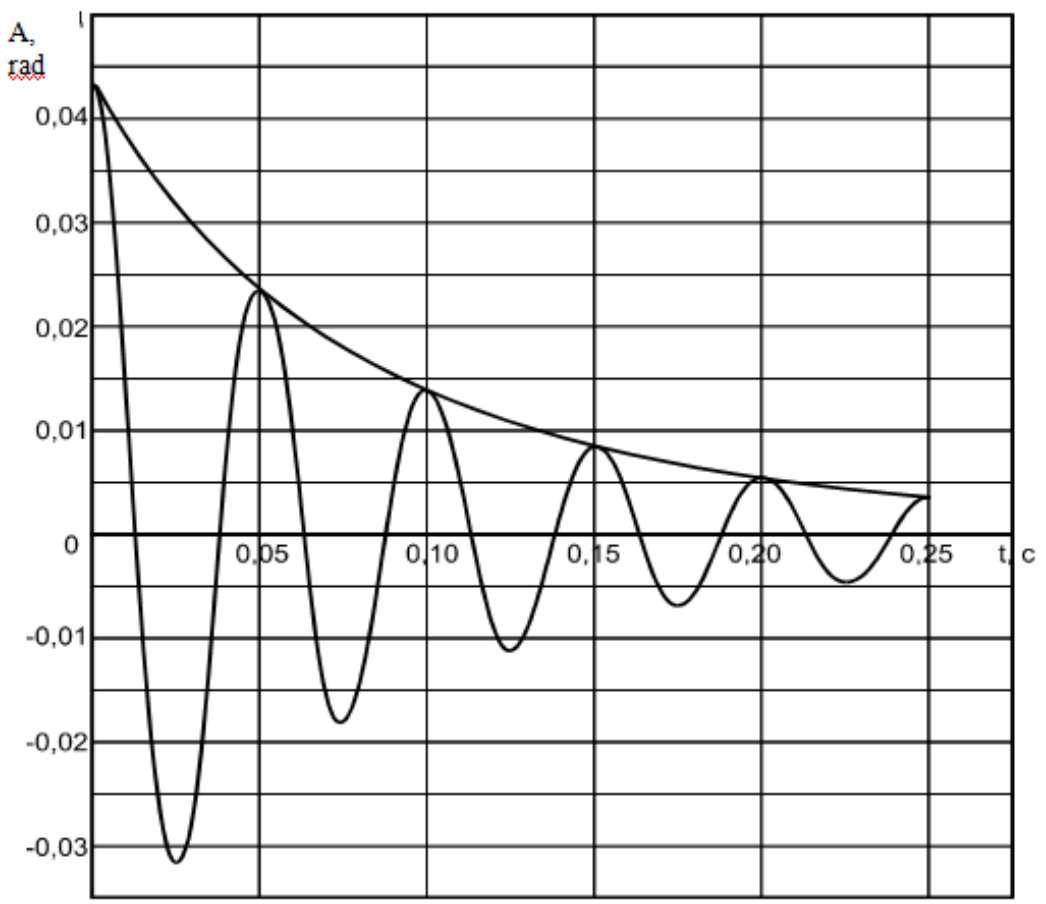

Fig. 2. Vibrogram of the oscillatory movement of the working body.

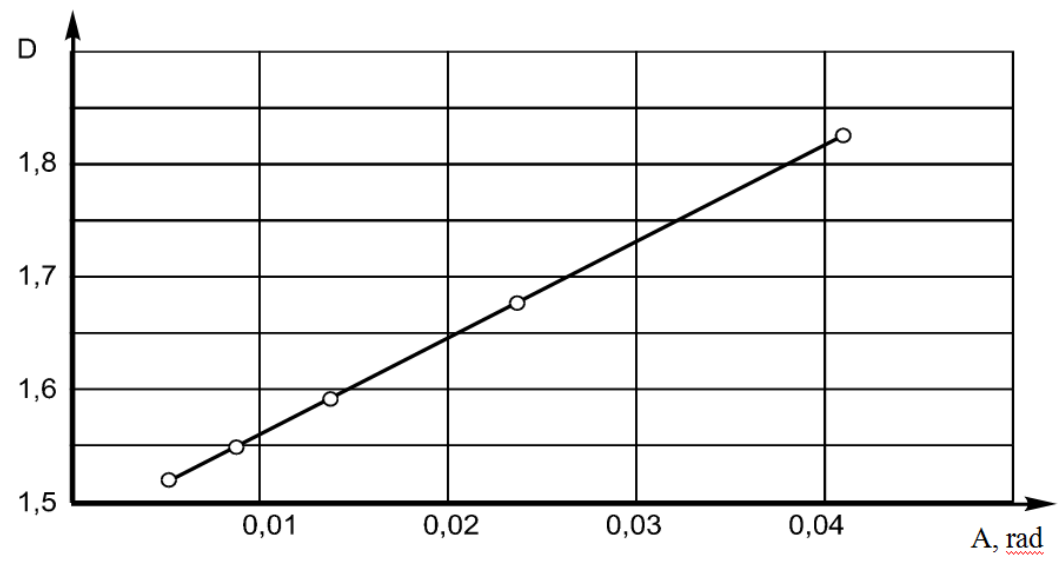

Fig. 3. Graph of the dependence of the decrement of oscillations on the amplitude.

Fig. 3 shows a graph of the dependence of the decrement of vibrations of the working body on the amplitude of vibrations. As can be seen from the figure, the greater the amplitude of the oscillations, the greater the decrement of the oscillations. This relationship is directly proportional. 


\section{Conclusions}

The equation (18) of motion of the blade of the working body of an earth-moving machine is obtained, depending on the technological properties of the soil and the design parameters of the self-oscillating working body. This equation can be used to determine the optimal parameters of the working bodies of earth-moving machines.

When determining the influence of the parameters of the working body and the ground on the nature of oscillatory movement of the blade, equation (18) is solved numerically.

As the solution of the resulting equation shows, the parameters of the working body and the properties of the soil affect such characteristics of the oscillatory motion as the frequency and period of vibrations, the decay decrement. At the same time, the greater the oscillation amplitude, the greater the attenuation decrement. This dependence is actually directly proportional.

\section{References}

1. Crowder Clifford D. Integral rippers for hydraulic excavator bucket, United States, E02F3/40; E02F3/96; E02F3/04; E02F3/40

2. David H. McNabb Ripper plough for soil tillage, United States Patent 172730, AA01B3920FI

3. Purser Ernest R. Ripper tooth attachment for a backhoe, E02F3/96; E02F3/04

4. Slaughter, James B. Adjustable ripper tooth for power shovels, United States Patent 3039210, E02F3/96; E02F3/04

5. V. L. Lapshin, Elastic-visco-plastic model for simulation of vibration separation processes, Mountain information and analytical Bulletin, 12, 285-288 (2008)

6. A method of regulating the parameters of vibration of the working bodies and the device for its implementation : patent 2231241 of the Rus. Federation; decl. 07.12.2001; publ. 27.09.2004. Bull. 27, 6

7. R. L. Sakhapov, R. V. Nikolaeva, M. H. Gatiyatullin, M. M. Makhmutov, Modeling the dynamics of the chassis of construction machines, Journal of Physics: Conference Series 738, 1, 012119 (2016) DOI: 10.1088/1742-6596/738/1/012119

8. A. I. Tretyakov, Analysis of design research in the vibrating working bodies of forestry of tillers, Voronezh scientific and technical Bulletin, 3(5), 90-101 (2013)

9. W. Batke, J. Siebeck, H. Halama, M. Ricci, F. Tintrup, Tooth cap for construction machinery, US Patent D463460, 2002.

10. V. A. Penchuk, Regularities of soil destruction by working bodies of machines for earthworks, Izvestiya VUZov. Construction, 1 (1999)

11. R. Sakhapov, M. Makhmutov, Influence of removable anti-skid device on the soil density, IOP Conference Series: Materials Science and Engineering, 786, 012021 (2020) DOI: $10.1088 / 1757-899 X / 786 / 1 / 012021$

12. I. M. Panov, Physical foundations of mechanics of soils (Phoenix, Kyiv, 266, 2008)

13. L. N. Kiseleva, V. N. Kuznetsova, The basic principles of mathematical modeling of the interaction of the working body of the digging machine with the developed soil/ Vestnik SibADI, 2, 5-9 (2011)

14. L. N. Kiseleva, Y. A. Fedotenko, Mathematical description of the process of interaction of the working bodies of mines, machine developed by the soil, Omsk scientific Bulletin of Omsk, 267-270 (2010)

15. L. N. Kiseleva, The influence of parameters chips mines, machines for traction calculation, Interuniversity collection of works of young scientists, postgraduates and students, 7, 246-250 (2010) 
16. R. L. Sakhapov, M. M. Makhmutov , M. M. Zemdikhanov, Experimental model of influence of moisture and soil type on shift resistivity, Izvestiya KGASU, 1(39), 286292 (2017)

17. R. L. Sakhapov, M. M. Makhmutov, Options of packages of different types of soils, Izvestiya KGASU, 2(32), 295-299 (2015)

18. D. Bruce, Earth working sweep, US Patent 20030010157, 2003.

19. Horst Konig, Maschinen im Baubetrieb (Wiesbaden:Vieweg+Teubner Verlag, 354, 2008) 